\title{
The coupling of a disk corona and a jet for the radio/X-ray correlation in black hole $\mathrm{X}$-ray binaries
}

\author{
Erlin Qiao \\ National Astronomical Observatories, Chinese Academy of Sciences, Beijing 100012, China \\ email: qiaoel@nao.cas.cn
}

\begin{abstract}
We interpret the radio/X-ray correlation of $L_{\mathrm{R}} \propto L_{\mathrm{X}}^{1.4}$ for $L_{\mathrm{X}} / L_{\mathrm{Edd}} \gtrsim 10^{-3}$ with a detailed disk corona-jet model, in which the accretion flow and the jet are connected by a parameter, $\eta$, describing the fraction of the matter in the accretion flow ejected outward to form the jet. We calculate $L_{\mathrm{R}}$ and $L_{\mathrm{X}}$ at different $\dot{M}$, adjusting $\eta$ to fit the observed radio/X-ray correlation of the black hole X-ray transient H1743-322 for $L_{\mathrm{X}} / L_{\mathrm{Edd}}>10^{-3}$. It is found that the value of $\eta$ for this radio/X-ray correlation for $L_{\mathrm{X}} / L_{\mathrm{Edd}}>10^{-3}$, is systematically less than that of the case for $L_{\mathrm{X}} / L_{\mathrm{Edd}}<10^{-3}$, which is consistent with the general idea that the jet is often relatively suppressed at the high luminosity phase in black hole X-ray binaries.
\end{abstract}

Keywords. accretion, accretion disk, X-rays: individual: H1743-322, X-rays: binaries, radio continuum: stars

\section{Introduction}

Recently, a growing number of black hole X-ray binaries (BHBs) have been discovered with a steeper radio/X-ray correlation of $L_{\mathrm{R}} \propto L_{\mathrm{X}} \sim^{1.4}$ for $L_{\mathrm{X}} \gtrsim 10^{-3} L_{\mathrm{Edd}}$, which is suggested to be explained within the framework of a radiatively efficient accretion flowjet model. Observationally, there is evidence of a coupling of the disk corona and jet at high mass accretion rates and a coupling of the radiatively inefficient accretion flow (RIAF) and jet at low mass accretion rates (Wu et al. 2013). Meanwhile, theoretically, for $\dot{M} \gtrsim \alpha^{2} \dot{M}_{\text {Edd }}$ (with $\alpha$ the viscosity parameter, $\dot{M}_{\text {Edd }}=1.39 \times 10^{18} M / M_{\odot} \mathrm{g} \mathrm{s}^{-1}$ ), the accretion flow will transit from a RIAF to a disk corona system (Qiao \& Liu 2009, 2010, 2013; Narayan \& Yi 1995b). In this work, we propose a disk corona-jet model to explain the radio/X-ray correlation of $L_{\mathrm{R}} \propto L_{\mathrm{X}}^{\sim^{1.4}}$ for $L_{\mathrm{X}} / L_{\mathrm{Edd}} \gtrsim 10^{-3}$. We briefly introduce the disk corona-jet model and the results in Section 2. Section 3 is the conclusion.

\section{The coupled disk corona-jet model and the result}

In the model, the disk and corona are radiatively and dynamically coupled (Liu et al. 2002, 2003). Thus, the energy fraction dissipated respectively in the corona and disk, and coronal density and temperature can all be self-consistently determined for given black hole mass and accretion rate. Then the spectrum emitted by the disk and corona can be calculated by Monte Carlo simulation. Assuming a fraction of matter in the accretion flow, $\eta \equiv \dot{M}_{\text {jet }} / \dot{M}$, is ejected to form the jet, we can also calculate the emergent spectrum from the jet. So far, the theoretical understanding of the jet formation is poor. Specifically, it is difficult to put constraints on the dependence of $\eta$ on $\dot{M}$ in our model, so we set $\eta$ as an independent parameter on $\dot{M}$ to fit the observations. 

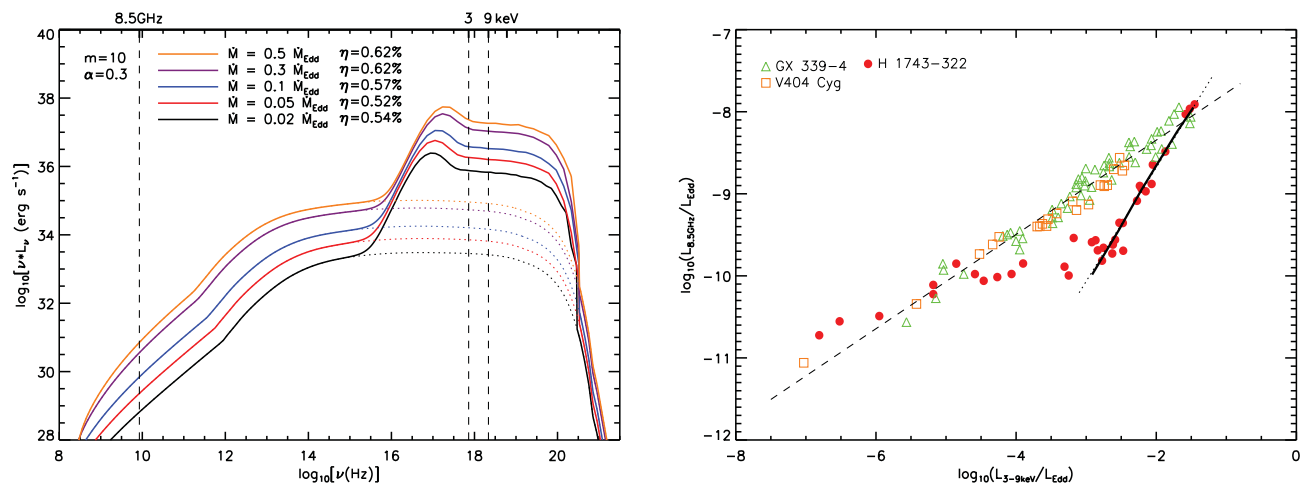

Figure 1. Left panel: Emergent spectra of the disk corona-jet model around a black hole with $M=10 M_{\odot}$ assuming $\alpha=0.3$ for modeling the radio/X-ray correlation of the black hole X-ray transient H1743-322 for $L_{3-9 \mathrm{keV}} / L_{\mathrm{Edd}}>10^{-3}$. From the bottom up, the solid lines are the combined emergent spectra of the disk corona-jet model for $\dot{M}=0.02,0.05,0.1,0.3$ and $0.5 \dot{M}_{\mathrm{Edd}}$, and the corresponding dotted lines are the emergent spectra from the jet with $\eta=0.54 \%, 0.52 \%, 0.57 \%, 0.62 \%$ and $0.62 \%$ respectively. Right panel: $L_{8.5 \mathrm{GHz}} / L_{\mathrm{Edd}}$ as a function of $L_{3-9 \mathrm{keV}} / L_{\mathrm{Edd}}$. The red $\bullet$ symbols are the observations for H1743-322, the green $\triangle$ symbols are the observations for GX 339-4, and orange $\square$ symbols are the observations for V404 Cyg. The dotted line is the best-fitting linear regression of H1743-322 for $L_{3-9 \mathrm{keV}} / L_{\mathrm{Edd}}>10^{-3}$. The dashed line is the best-fitting linear regression of GX 339-4 and V404 Cyg. The thick solid line is the model line, and the model spectra are shown in the left panel. The date are originally from Coriat et al. (2011).

We calculate $L_{\mathrm{R}}$ and $L_{\mathrm{X}}$ at different $\dot{M}$, adjusting $\eta$ to fit the observed radio/X-ray correlation of the black hole X-ray transient H1743-322 for $L_{\mathrm{X}} / L_{\mathrm{Edd}}>10^{-3}$. It is found that always the $\mathrm{X}$-ray emission is dominated by the disk corona and the radio emission is dominated by the jet. See Figure 1 for the details. We noted that the value of $\eta$ for the radio/X-ray correlation of $L_{\mathrm{R}} \propto L_{\mathrm{X}}{ }^{1.4}$ for $L_{\mathrm{X}} / L_{\mathrm{Edd}}>10^{-3}$, is systematically less than that of the case for $L_{\mathrm{X}} / L_{\mathrm{Edd}}<10^{-3}$, which is consistent with the general idea of jets often relatively suppressed at the high luminosity phase in black hole X-ray binaries.

\section{Conclusion}

We investigate the radio/X-ray correlation of $L_{\mathrm{R}} \propto L_{\mathrm{X}}^{1.4}$ for $L_{\mathrm{X}} / L_{\mathrm{Edd}} \gtrsim 10^{-3}$ within the framework of a disk corona-jet model. We note an interesting result, i.e., the fraction of the ejected matter $\eta(\sim 0.57 \%)$ for the radio/X-ray correlation of $L_{\mathrm{R}} \propto L_{\mathrm{X}}^{\sim 1.4}$ for $L_{\mathrm{X}} / L_{\mathrm{Edd}} \gtrsim 10^{-3}$, is systematically less than that of the case for $L_{\mathrm{X}} / L_{\mathrm{Edd}}<10^{-3}$ (at least $\eta \gtrsim 1 \%$ ), which may put some constraints on the jet formation, i.e., by suggesting that the strength of the jet power is relatively suppressed during the high luminosity phase in BHBs.

\section{References}

Coriat, M. et al., 2011, MNRAS, 414, 677

Liu, B. F. \& Mineshige, S., Ohsuga K., 2003, ApJ, 587, 571

Liu, B. F. \& Mineshige, S., Shibata K., 2002, ApJ, 572, L17

Narayan, R. \& Yi, I., 1995b, ApJ, 452, 710

Qiao, Erlin, Liu, B. F., 2009, PASJ, 61, 403

Qiao, Erlin, Liu, B. F., 2010, PASJ, 62, 661

Qiao, Erlin, Liu, B. F., 2013, ApJ, 764, 2

Wu, Q., Cao, X., Ho, Luis C., \& Wang, Ding-Xiong, 2013, ApJ, 770, 31 